\title{
Relationship of the expression levels of XIAP and p53 genes in hepatocellular carcinoma and the prognosis of patients
}

\author{
ZHIQIN LI, CHUNFANG HAN and JING FENG \\ Department of Infectious Diseases, Weifang People's Hospital, Weifang, Shandong 261041, P.R. China
}

Received May 24, 2017; Accepted July 19, 2017

DOI: $10.3892 / 01.2017 .6681$

\begin{abstract}
In this study, we measured mRNA and protein expression levels of X-linked inhibitor of apoptosis protein (XIAP) and p53 in hepatocellular carcinoma (HCC) and analyzed their relationships to clinicopathological parameters and the prognosis of the patients. Samples were obtained from tumors and tumor-adjacent normal tissues from 70 patients with HCC who were hospitalized in Weifang People's Hospital from January 2009 to December 2011. Quantitative polymerase chain reaction (qPCR) and immunohistochemistry (IHC) were used to detect the mRNA and protein expression levels, respectively. The clinical data of patients who were followed for 5 years from the day of the tumor-resection surgery were collected in detailed clinical histories. Statistical analyses were used to find relationships between the XIAP and p53 levels and the clinical variables and 5-year survival of patients. Our qPCR results showed that the mRNA expression levels of XIAP and p53 in HCC tumors were significantly higher than those in tumor-adjacent normal tissues. At the same time, IHC results showed that the positive expression rates of XIAP and p53 in HCC in tumors were $81.4 \%$ (57/70) and $72.9 \%(51 / 70)$, respectively and their high expression was related to invasion, metastasis and tumor staging. The overall 5-year survival rate of the patients was $15.7 \%$ (11/70). Single factor survival analysis showed that both XIAP and p53 were influencing factors of the overall survival rate of patients with HCC $(\mathrm{P}<0.01)$. In conclusion, high expression levels of XIAP and p53 are closely related to clinicopathological parameters of patients with HCC, especially related to invasion, metastasis and tumor staging. XIAP and p53 levels can be used as reference values to guide the treatment of HCC and estimate the prognosis.
\end{abstract}

Correspondence to: Dr Jing Feng, Department of Infectious Diseases, Weifang People's Hospital, 151 Guangwen, Kuiwen, Weifang, Shandong 261041, P.R. China

E-mail: feng_jingwf@163.com

Key words: hepatocellular carcinoma, X-linked inhibitor of apoptosis protein, $\mathrm{p} 53$, prognosis

\section{Introduction}

Hepatocellular carcinoma (HCC) is a common malignant tumor; its incidence ranks fifth and it has the third highest death rate among cancers (1). The pathogenesis of HCC is very complex; excessive alcohol drinking, viral hepatitis and nonalcoholic fatty liver have all been shown to induce carcinogenesis in normal liver cells (2-4). Studies on molecular physiology have found a dynamic balance between hepatocyte proliferation and apoptosis. When this balance is broken, apoptosis does not function well and tumor cell proliferation and even metastasis occur $(5,6)$.

The inhibitor of apoptosis (IAP) family includes protein factors that inhibit apoptosis in vivo (7). Among the many factors of the IAP family, the activity of the X-linked IAP protein (XIAP) is the strongest. XIAP is mainly composed of baculovirus IAP repeat (BIR) and zinc finger domains. BIR domains can inhibit the functions of caspase-3 and -7, while the zinc finger domains inhibit the functions of caspase-9; together these domains have the capacity for inhibiting all the caspases that induce apoptosis (8). Additionally, studies have also shown that XIAP plays a key role in other biological processes such as cell signal transduction and protein ubiquitination (9).

The p53 genes were some of the first factors shown to control cell proliferation and apoptosis, thus inhibiting tumors. However, mutations in the wild-type p53 gene can lead to inhibition of tumor cell apoptosis (10). Studies have confirmed that mutation of p53 gene happens in $50 \%$ of tumor tissues in humans, so p53 gene is used as tumor biomarker (11).

The present study was designed to measure expression of XIAP and $\mathrm{p} 53$ in HCC patients and to identify their relationships to clinicopathological parameters and prognosis of patients. Quantitative polymerase chain reaction (qPCR) and immunohistochemistry (IHC) were used to detect the expression levels of XIAP and p53 mRNA and proteins in HCC tumors and tumor-adjacent normal samples. Clinicopathological variables from the clinical history of 5 years from the day of surgery were used in the statistical analysis to calculate prognosis of patients.

\section{Materials and methods}

Specimens. Frozen and paraffin specimens of tumor tissues from 70 patients with $\mathrm{HCC}$ and the accompanying tumor 
adjacent normal tissues in 30 of those patients were used for the measurements. Patients admitted to Weifang People's Hospital (Weifang, China) from January 2009 to December 2011 were enrolled in the study. All patients were clinically and pathologically diagnosed with HCC and they all received surgical treatment for the first time and had no prior history of radiotherapy or chemotherapy. Patients with metastatic HCC, chronic hepatitis B or cirrhosis were excluded. In the end, there were 36 males and 34 females, aged 25-79 years with an average age of 47 . The Clinical Ethics Committee of our hospital approved all the procedures in the study and all participating patients or their family members signed the informed consent forms.

Detection of the expression levels of XIAP and p53 in specimens by $q P C R$. Approximately $50 \mathrm{mg}$ of frozen tumor tissues and tumor-adjacent tissues of patients were used to extract total RNA according to the method provided in the RNA isolation kit (Invitrogen Life Technologies, Carlsbad, CA, USA). The UV-Vis spectrophotometer (Hitachi, Ltd., Tokyo, Japan) was used to detect the total RNA concentration and purity (when the absorbance value ratio of A260/ A280 was 1.8-2.0, the RNA was deemed of good quality). cDNAs were obtained via reverse transcription according to the instructions in the reverse transcription kit (Invitrogen Life Technologies) and then the expression of XIAP and p53 mRNAs were detected following the method in the RT-PCR kit (Invitrogen Life Technologies) using cDNAs as templates. Glyceraldehyde 3-phosphate dehydrogenase (GAPDH) was used as an internal control. The primer sequences of XIAP, mutant p53 and GAPDH are shown in Table I, all primers were synthesized by Takara Bio (Dalian, China). The PCR conditions included $95^{\circ} \mathrm{C}$ for $10 \mathrm{~min}$, then a total of 40 cycles of 95 , 57 and $72^{\circ} \mathrm{C}$ for $30 \mathrm{sec}$ and then a final extension at $72^{\circ} \mathrm{C}$ for $5 \mathrm{~min}$. The relative expression levels were calculated using the $2^{-\Delta \mathrm{Cq}}$ method according to the following formula: $\Delta \mathrm{Cq}$ (target gene $)=\mathrm{Cq}$ (target gene) $-\mathrm{Cq}$ (control gene) .

Detection of the expression levels of XIAP and p53 proteins in patient specimens by IHC. The procedure was carried out according to the instructions of the IHC SP-9001 kit (Beijing Zhongshan Golden Bridge Biological Technology, Beijing, China). Briefly, after dewaxing of the paraffin sections, the endogenous peroxidase was inactivated with $3 \% \mathrm{H}_{2} \mathrm{O}_{2}$. The citrate buffer was used for thermal remediation and proteins were blocked with $10 \%$ goat serum. Primary rabbit polyclonal XIAP antibody (dilution, 1:500, cat. no. ab21278) and rabbit polyclonal p53 antibody (dilution, 1:500; cat. no. ab1431) were added and incubated at $4^{\circ} \mathrm{C}$ overnight. Then proteins were washed with phosphate-buffered saline (PBS) and secondary goat anti-rabbit (HRP) IgG antibody (dilution, 1:2,000; cat. no. ab6721) were added. All the antibodies were all purchased from Abcam (Cambridge, MA, USA). Under this condition, samples were incubated for $15 \mathrm{~min}$. After that, proteins were washed with PBS and diaminobenzidine solution was used for development in the dark. Then hematoxylin was used for restaining the samples. Finally, pictures were taken under the light microscope (TE2000-U; Nikon, Tokyo, Japan).

Three representative regions from each sample were selected and the results of immunohistochemical staining were
Table I. Primer sequences of qPCR.

\begin{tabular}{|c|c|}
\hline Gene & Primer sequence \\
\hline XIAP & $\begin{array}{l}\text { Forward 5'-AACCTTGTGATCGTGCCT-3' } \\
\text { Reverse 5'-ACCCTGGATACCATTTAGC-3' }\end{array}$ \\
\hline p53 & $\begin{array}{l}\text { Forward 5'-TGCGTGTGGAGTATTTGGATG-3' } \\
\text { Reverse 5'-TGGTACAGTCAGAGCCAACCTC-3' }\end{array}$ \\
\hline GAPDH & $\begin{array}{l}\text { Forward 5'-ATGGCACCGTCAAGGCTGAG-3' } \\
\text { Reverse 5'-GCAGTGATGGCATGGACTGT-3' }\end{array}$ \\
\hline
\end{tabular}

XIAP, X-linked inhibitor of apoptosis protein; GAPDH glyceraldehyde 3-phosphate dehydrogenase; qPCR, quantitative polymerase chain reaction.

evaluated by rating and grading the expression according to the staining intensity and the percentage of positive cells. The staining intensity was classified as stainless, brownish brown, tan and sepia (recorded as 0, 1, 2 and 3 points, respectively). In addition, the number of positive cells (magnification, $\mathrm{x} 400$ ) was classified as $<5,5-25,26-50$ and $>50 \%$ (recorded as $0,1,2$ and 3 points). In the end, the scores of the two indexes were added. Total points $>3$ were considered as positive for expression and $\leq 2$ as negative for expression. The final scores were used in the statistical analyses.

Relationship between the expression levels of XIAP and p53 in HCC tissues and the prognosis in patients. The 70 patients in the study were divided into a XIAP positive or a XIAP negative group and also into a p53-positive or -negative group, according to the expression levels of XIAP and p53 in HCC tissues. There were no differences in statistical significance in terms of age, sex, patient condition and other factors between the two groups (P $>0.05)$. The relationship of XIAP and p53 with clinicopathological parameters was analyzed using the available recorded clinical data of patients. All the 70 patients were followed up for 5 years and the follow-up rate was $100 \%$. The survival duration was recorded from the first day after surgery to the date of death of patients or the deadline of the follow-up. The statistical analysis was performed on a monthly basis.

Statistical analysis. SPSS 17.0 (IBM SPSS, Armonk, NY, USA) was used for statistical analyses in this study. Measurement data were expressed as mean \pm standard deviation and the t-test was used for comparisons between groups. The enumeration data were compared between the two groups using the $\chi^{2}$ test. Clinical prognostic data were calculated using the KaplanMeier survival analysis. $\mathrm{P}<0.05$ was considered to indicate a statistically significant difference.

\section{Results}

Detection of the expression levels of XIAP and p53 mRNAs in tissue specimens by qPCR. qPCR results (Fig. 1) showed that the expression levels of XIAP and p53 mRNAs in HCC tissues were significantly higher than those in tumor-adjacent normal tissues and the differences were statistically significant $(\mathrm{P}<0.01)$. 
A

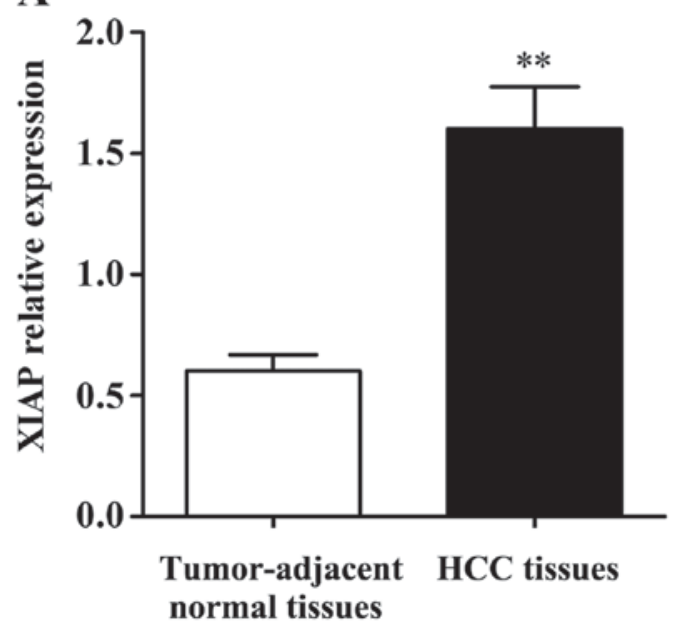

B

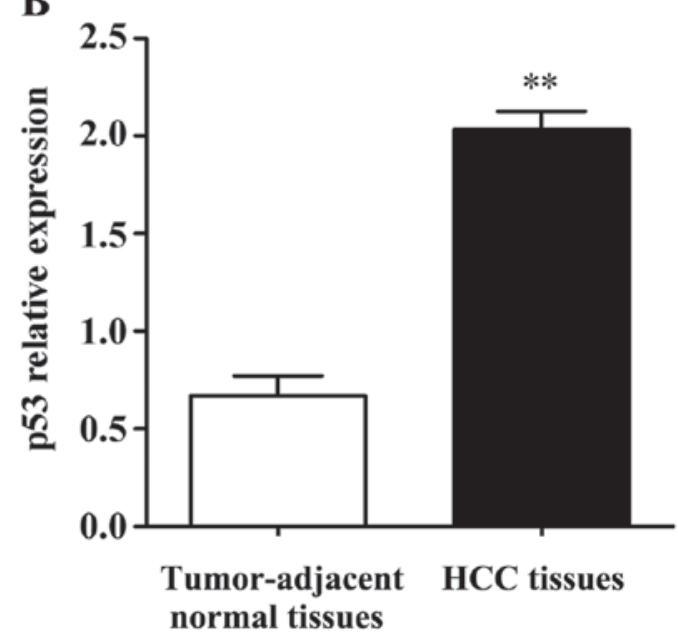

Figure 1. The expression levels of XIAP and p53 mRNAs in HCC tissues are significantly higher than those in tumor-adjacent normal tissues, ${ }^{* *} \mathrm{P}<0.01$. (A) The expression of XIAP mRNAs in tissue specimens. (B) The expression of p53 mRNAs in tissue specimens. XIAP, X-linked inhibitor of apoptosis protein; HCC, hepatocellular carcinoma.

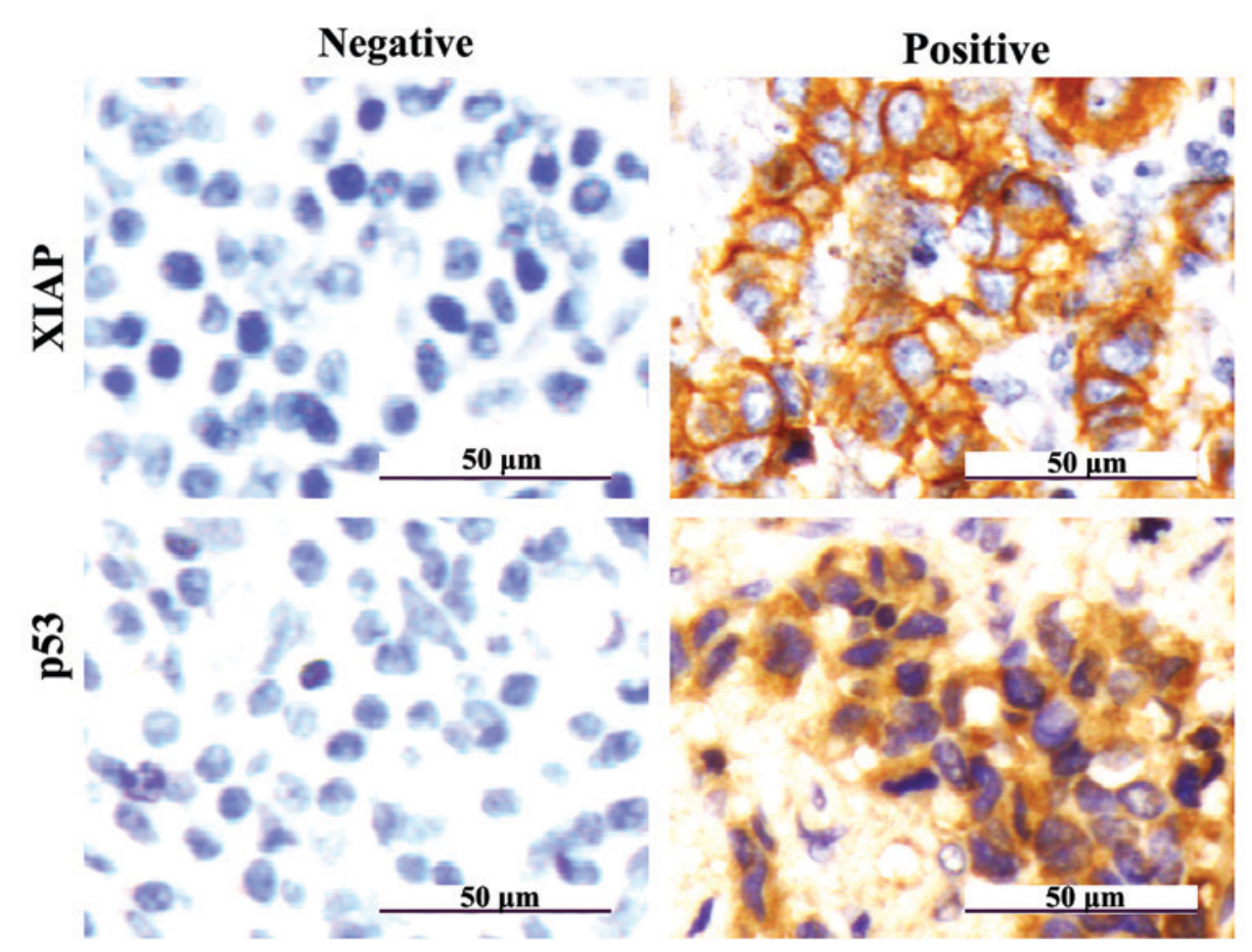

Figure 2. Detection of the expression of XIAP and p53 proteins in clinicopathological tissues by IHC. XIAP, X-linked inhibitor of apoptosis protein; IHC, immunohistochemistry.

Detection of the expression levels of XIAP and 553 proteins in clinicopathological tissues by IHC. In IHC results the color of stained XIAP and p53 by IHC was tan, with XIAP proteins mainly being present in the cytoplasm and partly in cell membranes and p53 proteins mainly present in the nucleus (Fig. 2).

The IHC scores were counted, the positive expression rates of XIAP in HCC and tumor-adjacent normal tissues were $81.4 \%$ (57/70) and $13.3 \%$ (4/30), respectively; and the difference was statistically significant $(\mathrm{P}<0.01)$. The positive expression rates of p53 in HCC and tumor-adjacent normal tissues were $72.9 \%(51 / 70)$ and $10.0 \%$ (3/30), respectively; and the difference was statistically significant $(\mathrm{P}<0.01)$ (Table II).

Relationship of clinicopathological parameters of HCC and the expression levels of XIAP and p53. The statistical analysis results showing the relationship between clinicopathological parameters of HCC patients and the expression levels of XIAP and p53 are shown in Table III and Fig. 2. The $\chi^{2}$ test showed that the positive expression of XIAP and p53 correlated with the occurrence of metastasis and higher tumor stage $(\mathrm{P}<0.01)$, but were not correlated with sex, age or tumor size $(\mathrm{P}>0.05)$. 
Table II. Positivity of expression of XIAP and p53 proteins in HCC and tumor-adjacent normal tissues.

\begin{tabular}{|c|c|c|c|c|c|c|c|}
\hline \multirow[b]{2}{*}{ Group } & \multirow[b]{2}{*}{ Case } & \multicolumn{3}{|c|}{ XIAP } & \multicolumn{3}{|c|}{ p53 } \\
\hline & & $\begin{array}{c}\text { Positive } \\
\text { expression }\end{array}$ & $\begin{array}{c}\text { Positive expression } \\
\text { rate }(\%)\end{array}$ & P-value & $\begin{array}{c}\text { Positive } \\
\text { expression }\end{array}$ & $\begin{array}{c}\text { Positive expression } \\
\text { rate }(\%)\end{array}$ & P-value \\
\hline HCC tissues & 70 & 57 & 81.4 & $<0.01$ & 51 & 72.9 & $<0.01$ \\
\hline $\begin{array}{l}\text { Tumor-adjacent } \\
\text { normal tissues }\end{array}$ & 30 & 4 & 13.3 & & 3 & 10.0 & \\
\hline
\end{tabular}

XIAP, X-linked inhibitor of apoptosis protein; HCC, hepatocellular carcinoma.

Table III. Relationship between high expression levels of XIAP and p53 with clinicopathological parameters of HCC patients.

\begin{tabular}{|c|c|c|c|c|c|c|c|}
\hline \multirow[b]{2}{*}{ Clinical data } & \multirow[b]{2}{*}{ Case } & \multicolumn{3}{|c|}{ XIAP } & \multicolumn{3}{|c|}{ p53 } \\
\hline & & $\begin{array}{c}\text { Positive } \\
\text { expression }\end{array}$ & $\begin{array}{c}\text { Positive expression } \\
\text { rate }(\%)\end{array}$ & P-value & $\begin{array}{c}\text { Positive } \\
\text { expression }\end{array}$ & $\begin{array}{c}\text { Positive expression } \\
\text { rate }(\%)\end{array}$ & P-value \\
\hline Sex & & & & & & & $>0.05$ \\
\hline Male & 36 & 30 & 83.3 & $>0.05$ & 26 & 72.2 & \\
\hline Female & 34 & 27 & 79.4 & & 25 & 73.5 & \\
\hline Age, years & & & & & & & $>0.05$ \\
\hline$\geq 50$ & 42 & 34 & 81.0 & $>0.05$ & 32 & 76.2 & \\
\hline$<50$ & 28 & 23 & 82.1 & & 19 & 67.9 & \\
\hline Tumor size & & & & & & & $>0.05$ \\
\hline$\geq 5 \mathrm{~cm}$ & 39 & 31 & 79.5 & $>0.05$ & 28 & 71.8 & \\
\hline$<5 \mathrm{~cm}$ & 31 & 26 & 83.9 & & 23 & 74.2 & \\
\hline Invasion and metastasis & & & & & & & $<0.01$ \\
\hline Yes & 46 & 43 & 93.5 & $<0.01$ & 41 & 89.1 & \\
\hline No & 24 & 14 & 58.3 & & 10 & 41.7 & \\
\hline Tumor staging & & & & & & & $<0.01$ \\
\hline I-II & 26 & 15 & 57.7 & $<0.01$ & 11 & 42.3 & \\
\hline III-IV & 44 & 42 & 95.5 & & 40 & 90.9 & \\
\hline
\end{tabular}

XIAP, X-linked inhibitor of apoptosis protein; HCC, hepatocellular carcinoma.

Table IV. Single factor analysis of the relationship of the expression levels of XIAP and p53 with the overall survival rate of HCC patients.

\begin{tabular}{lcccc}
\hline Group & Case & 5-year survival case & 5-year survival rate (\%) & Wald (log-rank) \\
\hline XIAP & & & & P-value \\
Positive & 57 & 5 & 8.8 & 15.14 \\
Negative & 13 & 6 & 76.2 & $<0.01$ \\
p53 & & 4 & 36.8 & 15.68 \\
Positive & 51 & 7 & & $<0.01$ \\
Negative & 19 & 4.8 & \\
\hline
\end{tabular}

XIAP, X-linked inhibitor of apoptosis protein; HCC, hepatocellular carcinoma.

Single factor analysis of the survival time and prognosis of patients. A total of 70 patients with HCC were followed up for a maximum of 5 years. The number of 5-year survivors was 11 and the number of deaths was 59, as shown in Table IV. 

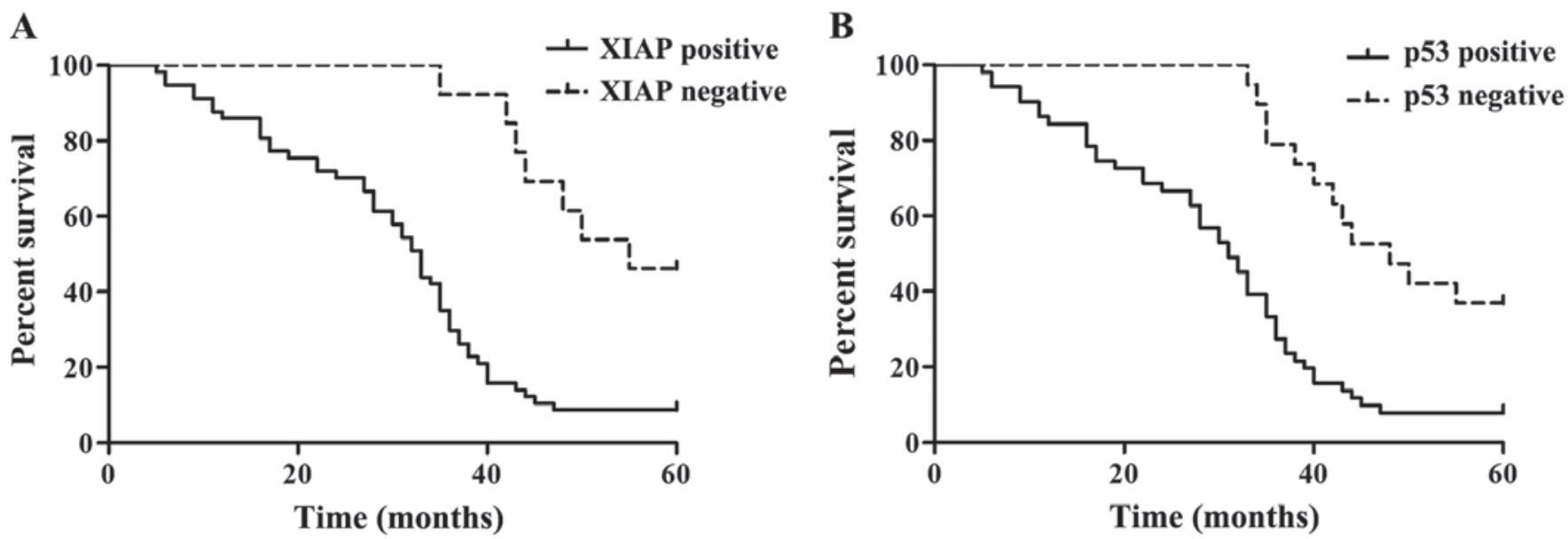

Figure 3. The expression of XIAP and p53 and Kaplan-Meier survival curve of patients with HCC. HCC patients with negative expression of XIAP and p53 have better survival prognoses. (A) The Kaplan-Meier survival curves of HCC patients with positive and negative expression of XIAP. (B) The KaplanMeier survival curves of HCC patients with positive and negative expression of p53. XIAP, X-linked inhibitor of apoptosis protein; HCC, hepatocellular carcinoma.

Fig. 3 shows the Kaplan-Meier survival curves of 70 patients with different expression of XIAP and p53. The results showed that HCC patients with negatively expressed XIAP and p53 had a relatively better survival prognosis. Differences in the overall survival curve were analyzed using the log-rank test, as shown in Table III. The results of single factor survival analysis showed that the effects of XIAP and p53 on the overall survival rate of patients with $\mathrm{HCC}$ were statistically significant $(\mathrm{P}<0.01)$.

\section{Discussion}

$\mathrm{HCC}$ is one of the most common malignant tumors in China (12). Presently, more than 100 oncogenes have been found in studies, but the number of tumor suppressor genes is very small. Mutations, deletions and inactivations of tumor suppressor genes are events closely related to the occurrence and development of tumors (13).

XIAP, as an important member of the IAP family, can specifically inhibit the activity of caspase-3, -7 and -9, and inhibit the apoptosis of tumor cells (14). Clinical studies have shown that XIAP expression levels are significantly higher in a variety of malignant tumor tissues than in normal tissues (15).

The p53 gene is located on human chromosome 17p13 and is a kind of tumor suppressor and pro-apoptosis gene. Wild-type p53 plays a key role in cell gene transcription, cell cycle regulation, apoptosis, proliferation and differentiation $(16,17)$. Studies have found that p53 gene mutations are closely related to the occurrence and development of HCC $(18,19)$. The amount of wild-type p53 proteins is low in normal cells and their half-life periods are short, so IHC and other methods cannot detect them. However, the half-life periods of mutant p53 proteins are relatively longer and the amounts are relatively higher, allowing for their detection $(20,21)$. Studies have found that mutant p53 proteins are highly expressed in tumor tissues of patients with breast cancer and the degree of malignancy of the cancer in these patients is high resulting in poor prognosis after surgery (22).
In order to find if tumor tissue levels of XIAP and p53 in HCC patients are also useful for prognoses, the expressions of XIAP and p53 mRNAs in tumor tissues of HCC patients were detected by qPCR. Our results showed the expression levels of XIAP and p53 mRNAs in HCC tissues were significantly higher than those in tumor-adjacent normal tissues. Furthermore, the IHC results showed that the positive expression rates of XIAP and $\mathrm{p} 53$ proteins in HCC tissues were $81.4 \%(57 / 70)$ and $72.9 \%$ (51/70), respectively, which were significantly higher than those in tumor-adjacent normal tissues. A further analysis, in combination with the clinicopathological features of patients, revealed that the expression of XIAP and p53 was related to the occurrence of metastasis and tumor staging; but not related to sex, age or the tumor size. Single factor Kaplan-Meier survival analysis was used to analyze the effects of different mRNA expression levels of XIAP and p53 on the overall survival rate curve of patients. The results showed the overall survival period of HCC patients with high expression levels of XIAP and p53 was relatively short.

A large number of experiments have shown that XIAP and p53 are closely related to the occurrence and development of a variety of tumors. A study found that XIAP protein levels in kidney clear cell carcinoma tissue specimens were high and the expression levels in the advanced stages were significantly higher than those in the early stages (23). Another study found the expression levels of p53 in 62 cases of primary $\mathrm{HCC}$ to be higher in cancer tissues than those in tumor-adjacent normal tissues (24). Our findings corroborate these studies and further confirmed that XIAP and p53 are highly expressed in $\mathrm{HCC}$, and the expression of XIAP and p53 were related to the metastasis, staging and prognosis of HCC patients.

In conclusion, the positive expression of XIAP and p53 are closely related to the occurrence and development of HCC, especially to tumor metastasis and tumor staging. XIAP and p53 can be used as reference markers to guide the treatment of HCC and estimate its prognosis. 


\section{References}

1. Yang JD and Roberts LR: Epidemiology and management of hepatocellular carcinoma. Infect Dis Clin North Am 24: 899-919, 2010.

2. Duan XY, Zhang L, Fan JG and Qiao L: NAFLD leads to liver cancer: Do we have sufficient evidence? Cancer Lett 345: 230-234, 2014

3. Karagozian R, Derdák Z and Baffy G: Obesity-associated mechanisms of hepatocarcinogenesis. Metabolism 63: 607-617, 2014.

4. Nakagawa H, Umemura A, Taniguchi K, Font-Burgada J, Dhar D, Ogata H, Zhong Z, Valasek MA, Seki E, Hidalgo J, et al: ER stress cooperates with hypernutrition to trigger TNF-dependent spontaneous HCC development. Cancer Cell 26: 331-343, 2014

5. Parkin DM, Bray F, Ferlay J and Pisani P: Global cancer statistics, 2002. CA Cancer J Clin 55: 74-108, 2005.

6. Han XJ, Dong BW, Liang P, Yu XL and Yu DJ: Local cellular immune response induced by ultrasound-guided tumor bed superantigen injection after percutaneous microwave coagulation therapy for liver cancer. Zhonghua Zhong Liu Za Zhi 31: 602-606, 2009 (In Chinese).

7. Deveraux QL and Reed JC: IAP family proteins - suppressors of apoptosis. Genes Dev 13: 239-252, 1999.

8. Holcik M, Gibson H and Korneluk RG: XIAP: Apoptotic brake and promising therapeutic target. Apoptosis 6: 253-261, 2001.

9. Liston P, Fong WG and Korneluk RG: The inhibitors of apoptosis: There is more to life than Bcl2. Oncogene 22: 8568-8580, 2003.

10. Maniwa Y, Yoshimura M, Obayashi C, Inaba M, Kiyooka K Kanki $\mathrm{M}$ and Okita Y: Association of p53 gene mutation and telomerase activity in resectable non-small cell lung cancer. Chest 120: 589-594, 2001.

11. Graziano SL, Tatum A, Herndon JE II, Box J, Memoli V, Green MR and Kern JA: Use of neuroendocrine markers, p53 and HER 2 to predict response to chemotherapy in patients with stage III non-small cell lung cancer: A cancer and leukemia group B study. Lung Cancer 33: 115-123, 2001.

12. Green DR and Kroemer G: Cytoplasmic functions of the tumour suppressor p53. Nature 458: 1127-1130, 2009.

13. Song LP, Li YP, Wang N, Li WW, Ren J, Qiu SD, Wang QY and Yang GX: NT4(Si)-p53(N15)-antennapedia induces cell death in a human hepatocellular carcinoma cell line. World J Gastroenterol 15: 5813-5820, 2009.
14. Hu W, Wang F, Tang J, Liu X, Yuan Z, Nie C and Wei Y: Proapoptotic protein Smac mediates apoptosis in cisplatinresistant ovarian cancer cells when treated with the antitumor agent AT101. J Biol Chem 287: 68-80 2012.

15. Hussain AR, Uddin S, Ahmed M, Bu R, Ahmed SO, Abubaker J, Sultana M, Ajarim D, Al-Dayel F, Bavi PP, et al: Prognostic significance of XIAP expression in DLBCL and effect of its inhibition on AKT signalling. J Pathol 222: 180-190, 2010.

16. Shangary S and Wang S: Targeting the MDM2-p53 interaction for cancer therapy. Clin Cancer Res 14: 5318-5324, 2008.

17. Shangary S, Qin D, McEachern D, Liu M, Miller RS, Qiu S, Nikolovska-Coleska Z, Ding K, Wang G, Chen J, et al: Temporal activation of p53 by a specific MDM2 inhibitor is selectively toxic to tumors and leads to complete tumor growth inhibition. Proc Natl Acad Sci USA 105: 3933-3938, 2008.

18. Scoumanne A and Chen X: Protein methylation: A new mechanism of p53 tumor suppressor regulation. Histol Histopathol 23: 1143-1149, 2008.

19. Lu C and El-Deiry WS: Targeting p53 for enhanced radio- and chemo-sensitivity. Apoptosis 14: 597-606, 2009.

20. Hall PA, Ray A, Lemoine NR, Midgley CA, Krausz T and Lane DP: p53 immunostaining as a marker of malignant disease in diagnostic cytopathology. Lancet 338: 513, 1991.

21. Martinazzi M, Crivelli F, Zampatti C and Martinazzi S: Relationship between p53 expression and other prognostic factors in human breast carcinoma. An immunohistochemical study. Am J Clin Pathol 100: 213-217, 1993.

22. Chen W, Li J, Liu C, Chen X, Zhu Y, Yang Y, Gong Y, Wang T, Miao X and Nie X: A functional p53 responsive polymorphism in KITLG, rs4590952, does not affect the risk of breast cancer. Sci Rep 4: 6371, 2014

23. Ramp U, Krieg T, Caliskan E, Mahotka C, Ebert T, Willers R, Gabbert HE and Gerharz CD: XIAP expression is an independent prognostic marker in clear-cell renal carcinomas. Hum Pathol 35: 1022-1028, 2004.

24. Hong Y, Zhong R and Hang Z: The association study and its clinical significance of the expression of P53, AR and ER in the primary hepatocelluar carcinoma (liver cancer) and surrounding tissue. Int Med Health Guid News 12: 12-14, 2006. 